\title{
LAGRANGIAN FLOER HOMOLOGY ON SYMPLECTIC BLOW UPS
}

\author{
ANDRÉS PEDROZA
}

\begin{abstract}
We show how to compute the Lagrangian Floer homology in the onepoint blow up of the proper transform of Lagrangians submanifolds, solely in terms of information of the base manifold. As an example we present an alternative computation of the Lagrangian quantum homology in the one-point blow up of $\left(\mathbb{C} P^{2}, \omega_{F S}\right)$ of the proper transform of the Clifford torus.
\end{abstract}

\section{INTRODUCTION}

Lagrangian Floer homology (LFH for short) is a powerful tool developed by A. Floer [8, 7] to solve the Arnol'd Conjecture about the minimal number of fixed points of a Hamiltonian diffeomorphism on a closed symplectic manifold. Nevertheless, LFH is important in its own right due to its rich algebraic structure and for its interference in the classification of Lagrangian submanifolds under Hamiltonian equivalence, among other things. Unfortunately, it is extremely difficult to compute the LFH of a pair of Lagrangian submanifolds.

The aim of this article is to understand how the LFH changes after symplectically blowing up a point that does not lie in the Lagrangian submanifolds. Further, we show how to compute the differential in the blown up manifold from the differential in the base manifold and hence the LFH in the symplectic one-point blow up using solely data from the base symplectic manifold. We used LFH for monotone Lagrangian submanifolds as defined by Y.-G. Oh in [15, 16] and also the quantum homology of a Lagrangian as defined by P. Biran and O. Cornea in [4] that was previously defined also by Y.-G. Oh in [17]. Hence, $(M, \omega)$ will stand for a symplectic manifold that is either closed or convex at infinity and the Lagrangian submanifolds will be assumed to be monotone with minimal Maslov number greater than or equal to two.

As for the Lagrangian submanifolds, beside the monotonicity condition we impose another condition which is directly related to the definition of the symplectic onepoint blow up. Recall that $L \subset(M, \omega)$ is called monotone if there exists a positive number $\lambda$ such that $\mu_{L}=\lambda \cdot \omega$ on $\mathrm{H}_{2}(M, L)$. In order to remain in the context of monotone Lagrangians, the weight $\rho$ of the one-point blow up must be equal to

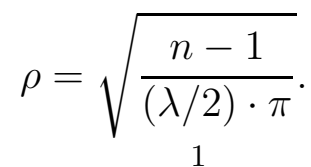


Accordingly we focus on symplectic manifolds whose Gromov's width is such that $c(M, \omega)>2(n-1) / \lambda$. Thus, if $\iota:\left(B^{2 n}(\rho), \omega_{0}\right) \rightarrow(M, \omega)$ is the symplectic embedding used to define the symplectic one-point blow up $\left(\widetilde{M}, \widetilde{\omega}_{\rho}\right)$, we restrict our analysis to monotone Lagrangian submanifolds $L \subset(M, \omega)$ such that $L \cap \iota\left(B^{2 n}(\rho)\right)$ is empty. Here $B^{2 n}(\rho) \subset\left(\mathbb{R}^{2 n}, \omega_{0}\right)$ is the closed ball of radius $\rho$ centred at the origin. This means that the Lagrangian submanifold that we consider are such that $c(M \backslash L, \omega)>2(n-1) / \lambda$.

This condition is quite restrictive. Here is an example that shows our limitation. Consider $\left(\mathbb{C} P^{n}, \omega_{\mathrm{FS}}\right)$ where the symplectic form is normalized so that the symplectic area of the line is $\pi$. Then $\mathbb{R} P^{n} \subset\left(\mathbb{C} P^{n}, \omega_{\mathrm{FS}}\right)$ is a monotone Lagrangian submanifold with minimal Maslov number $n+1$. For $n>2$, the Lagrangian $\mathbb{R} P^{n}$ fails the condition to be away from the prescribed embedded ball. For, according to P. Biran [2, Theorem 1.B], the image of any symplectic embedding $\left(B^{2 n}(\rho), \omega_{0}\right) \rightarrow\left(\mathbb{C} P^{n}, \omega_{\mathrm{FS}}\right)$ with $\rho^{2} \geq 1 / 2$ intersects the Lagrangian $\mathbb{R} P^{n}$. On the other hand, the value of the weight of the blow up, in order to obtain a monotone Lagrangian, must be equal to $\rho^{2}=(n-1) /(n+1)$. Hence, for $n>2$ the monotone Lagrangian $\mathbb{R} P^{n} \subset\left(\mathbb{C} P^{n}, \omega_{\mathrm{FS}}\right)$ fails the condition to be disjoint from the embedded ball used to define the symplectic one-point blow up.

Let $\pi:\left(\widetilde{M}, \widetilde{\omega}_{\rho}\right) \rightarrow(M, \omega)$ denote the blow up map. From now on, we always assume that the weight of the blow up is such that $\left(\widetilde{M}, \widetilde{\omega}_{\rho}\right)$ is monotone and the Lagrangian submanifolds are such that the Gromov's width of their complement is greater than $2(n-1) / \lambda$. Recall that away from the preimage of the embedded ball $\iota\left(B^{2 n}(\rho)\right), \pi$ is a symplectic diffeomorphism. Hence if $L \subset(M, \omega)$ is a Lagrangian submanifold such that $L \cap \iota\left(B^{2 n}(\rho)\right)=\emptyset$, then the proper transform $\pi^{-1}(L)$ is a Lagrangian submanifold in $\left(\widetilde{M}, \widetilde{\omega}_{\rho}\right)$. Let $\widetilde{L} \subset\left(\widetilde{M}, \widetilde{\omega}_{\rho}\right)$ denote the Lagrangian submanifold $\pi^{-1}(L)$. If $L$ is monotone, it follows from Lemma 4.5 that $\widetilde{L}$ is also monotone. However their minimal Maslov number do not have to coincide. In general the minimal Maslov number of $\widetilde{L}$ decreases in comparison with the minimal Maslov number of $L, N_{\widetilde{L}} \leq N_{L}$.

For instance consider $L=\mathbb{R} P^{2}$ in $\left(\mathbb{C} P^{2}, \omega_{\mathrm{FS}}\right)$. Thus $L$ is monotone and its minimal Maslov number is three. Further, there is a symplectic embedding of $\left(B^{4}(1 / \sqrt{3}), \omega_{0}\right)$ into $\left(\mathbb{C} P^{2}, \omega_{\mathrm{FS}}\right)$ whose image does not intersect $L$. Let $A \in \mathrm{H}_{2}\left(\mathbb{C} P^{2}, \mathbb{R} P^{2}\right)$ be such that $\mu_{L}(A)=3$ and $\widetilde{A} \in \mathrm{H}_{2}\left(\widetilde{\mathbb{C} P^{2}}, \widetilde{L}\right)$ the proper transform of $A$. By the definition of the Maslov index if follows that $\mu_{\widetilde{L}}(\widetilde{A})=3$. If $L_{E} \in \mathrm{H}_{2}\left(\widetilde{\mathbb{C} P}{ }^{2}\right)$ is the class of the exceptional line, then $c_{1}\left(\widetilde{\mathbb{C} P}{ }^{2}\right)\left(L_{E}\right)=-1$ and

$$
\mu_{\widetilde{L}}\left(\widetilde{A} \# L_{E}\right)=\mu_{\widetilde{L}}(\widetilde{A})+2 c_{1}\left(\widetilde{\mathbb{C} P}^{2}\right)\left(L_{E}\right)=1 .
$$

That is $N_{\mathbb{R} P^{2}}=1$. In particular its LFH is not well-defined in this case.

Definition 1.1. Let $(M, \omega)$ be a symplectic manifold of dimension $2 n \geq 4$ that is either closed or symplectically convex at infinity. A collection of closed Lagrangian submanifolds $L_{0}, \ldots, L_{k} \subset(M, \omega)$ is called admissible in $(M, \omega)$ if 
a) the Lagrangians are monotone with the same monotonicity constant $\lambda$,

b) $c\left(M \backslash\left(L_{0} \cup \cdots \cup L_{k}\right), \omega\right)>2(n-1) / \lambda$ and

c) $N_{\widetilde{L}_{j}} \geq 2$ for $j \in\{1,2 \ldots, k\}$ where the one-point blow up of $(M, \omega)$ is taken with respect to any symplectic embedding of the ball in $M \backslash\left(L_{0} \cup \cdots \cup L_{k}\right)$ whose radius is given by (11).

Consider $L_{0}, L_{1} \subset(M, \omega)$ Lagrangians submanifolds that are admissible. The goal of this note is to compute $\operatorname{HF}\left(\widetilde{L}_{0}, \widetilde{L}_{1}\right)$ in terms of the based manifold $(M, \omega)$, the Lagrangians $L_{0}$ and $L_{1}$, and the Floer complex $\left(\mathrm{CF}\left(L_{0}, L_{1}\right), \partial\right)$. (As mentioned above, the pearl complex will also be considered.) The first step is to understand the role played by the blown up point. The blown up point not only has to lie in the complement of the Lagrangian submanifolds, it must be a generic point. The reason been that any pair of points in the complement of $L_{0} \cup L_{1} \subset(M, \omega)$ can be mapped one to the other by a Hamiltonian diffeomorphism that leaves the Lagrangian submanifolds fixed.

Theorem 1.2. Let $L_{0}, L_{1} \subset(M, \omega)$ be admissible Lagrangian submanifolds. Assume that there exists $J \in \mathcal{J}_{\text {reg }}\left(L_{0}, L_{1}\right)$ such that $\iota^{*} J=J_{0}$ where $\iota:\left(B^{2 n}(\rho), \omega_{0}\right) \rightarrow(M, \omega)$ is a symplectic embedding that avoids the Lagrangian submanifolds and $\rho$ is given by Eq. (11). If $\pi_{j}:\left(\widetilde{M}_{j}, \widetilde{\omega}_{j}\right) \rightarrow(M, \omega)$ are the monotone one-point blow up of $(M, \omega)$ at $x_{j} \in M \backslash\left(L_{0} \cup L_{1}\right)$ for $j=1,2$, then

$$
\mathrm{HF}_{*}\left(\pi_{1}^{-1}\left(L_{0}\right), \pi_{1}^{-1}\left(L_{1}\right)\right) \simeq \mathrm{HF}_{*}\left(\pi_{2}^{-1}\left(L_{0}\right), \pi_{2}^{-1}\left(L_{1}\right)\right)
$$

as $\Lambda$-modules.

Remark. The hypothesis on the almost complex structure, $\iota^{*} J=J_{0}$, is required in order to move holomorphic information from the based manifold to the blow up manifold and vice versa. Here, $J_{0}$ stands for the standard complex structure on $\mathbb{C}^{n}$. Actually, is not hard to obtain an almost complex structure $J$ such that satisfies $\iota^{*} J=J_{0}$. The problem is to guarantee that such almost complex structure is regular. Although, $\mathcal{J}_{\text {reg }}\left(L_{0}, L_{1}\right)$ is a dense set in $\mathcal{J}(M, \omega)$ the set $\left\{J \in \mathcal{J}(M, \omega) \mid \iota^{*} J=J_{0}\right\}$ is closed and has a dense complement. On the other side, most known examples of symplectic manifolds that at the same time are complex manifolds, the complex structure happens to be regular.

Hence $\operatorname{HF}_{*}\left(\widetilde{L}_{0}, \widetilde{L}_{1}\right)$ is independent of the blown up point of $(M, \omega)$ in the complement of $L_{0} \cup L_{1}$. Now that the lack of relevance of the blown up point has been settled, we focus con the comparison of the LFH on the based manifold and the one-point blow up. By the observation that the blow up map is a symplectic diffeomorphism away from the embedded ball, heuristically $\operatorname{HF}_{*}\left(L_{0}, L_{1}\right)$ is isomorphic to $\operatorname{HF}_{*}\left(\widetilde{L}_{0}, \widetilde{L}_{1}\right)$ if the blown up point is not implicated in the data involved in the definition of $\operatorname{HF}_{*}\left(L_{0}, L_{1}\right)$. The precise statement is the following. 
Theorem 1.3. Let $L_{0}, L_{1} \subset(M, \omega)$ be admissible Lagrangians. If there exists $x_{0} \in$ $M \backslash\left(L_{0} \cup L_{1}\right)$ and $J \in \mathcal{J}_{\text {reg }}\left(L_{0}, L_{1}\right)$ such that $\iota^{*} J=J_{0}$; where $\iota$ is the symplectic embedding of the ball, and $x_{0}$ does not lie in any J-holomorphic strip of MaslovViterbo index $2 n-1$, then

$$
\mathrm{HF}_{*}\left(\widetilde{L}_{0}, \widetilde{L}_{1}\right) \simeq \mathrm{HF}_{*}\left(L_{0}, L_{1}\right)
$$

as $\Lambda$-modules.

Remark. - A straightforward class of examplea of the above result is the case when the symplectic manifold is non compact. For instance $\left(\mathbb{C}^{n}, \omega_{0}\right)$ and any pair of compact Lagrangian submanifolds that meet the hypothesis of Theorem 1.3. That is, after blowing up one point in $\left(\mathbb{C}^{n}, \omega_{0}\right)$, the induced compact Lagrangian submanifolds in $\left(\widetilde{\mathbb{C}}^{n}, \widetilde{\omega}_{0}\right)$ will continue to be displaceable.

- The reason of the value of $2 n-1$ of the Maslov-Viterbo index of the holomorphic strip is the following. The image of $\mathbb{R} \times[0,1] \sqrt{-1}$ under the set of all $J$-holomorphic strips in the same class of Maslov-Viterbo index $2 n-1$, generically is $2 n$-dimensional and after blowing up it could induced a holomorphic strip on the one-point blow up of Maslov-Viterbo index 1. This claim is proved in Proposition 4.6. Basically, this is the only possibility to alter the differential of the Floer complex of the blow up in comparison with the differential of the based manifold.

- By Theorem 1.2, the LFH is independent of the blown up point, thus some uniformity on the complement of $L_{0} \cup L_{1}$ is required in order to alter the Floer differential. This will be done below by assuming a uniruled condition in the context of quantum homology.

From Theorem 1.2, $\operatorname{HF}_{*}\left(\widetilde{L}_{0}, \widetilde{L}_{1}\right)$ is independent of the point in $(M, \omega)$ that is blown up. Further, from Theorem 1.3 holomorphic strips of Maslov-Viterbo index $2 n-1$ are the strips that can affect the differential in the blown up manifold in comparison with the differential in the base manifold. Thus a necessary condition for $\operatorname{HF}_{*}\left(\widetilde{L}_{0}, \widetilde{L}_{1}\right)$ to be non isomorphic to $\operatorname{HF}_{*}\left(L_{0}, L_{1}\right)$ is the following: for any $x_{0} \in M \backslash\left(L_{0} \cup L_{1}\right)$ there exists a dense set $\mathcal{J}_{\text {reg }}\left(L_{0}, L_{1}\right)$ of regular almost complex structures such that for every $J \in \mathcal{J}_{\text {reg }}\left(L_{0}, L_{1}\right)$ there exists a $J$-holomorphic strip $u: \mathbb{R} \times[0,1] \sqrt{-1} \rightarrow M$ of Maslov-Viterbo index $2 n-1$ such that $x_{0} \in u(D)$ with the usual boundary conditions. In this paper we do not explore this condition in general. Instead we consider the case $L_{0}=L_{1}$. Thus from now on, we only consider the case $\operatorname{HF}_{*}(L, L)$. Further, in order to avoid the Hamiltonian perturbation that is involved in the calculation of $\operatorname{HF}_{*}(L, L)$ and present a clear picture of the phenomenon implicated in our computation we used quantum homology $\mathrm{QH}_{*}(L)$ instead of $\mathrm{HF}_{*}(L, L)$. Thus $\Lambda$ stands for two different rings depending it $\mathrm{HF}_{*}(L, L)$ or $\mathrm{QH}_{*}(L)$ is being used. 
From Theorem 1.2, $\mathrm{QH}_{*}(\widetilde{L})$ is independent of the point in $(M, \omega)$ that is blown up. Further, the condition that $x_{0}$ does not lie in any $J$-holomorphic strip of MaslovViterbo index $2 n-1$ of Theorem 1.3 is replace by the condition that $x_{0}$ does not lie in any $J$-holomorphic disk of Maslov index $2 n$. In this case, $\mathrm{QH}_{*}(\widetilde{L}) \simeq \mathrm{QH}_{*}(L)$. Henceforth, pearly trajectories that contain a $J$-holomorphic disk of Maslov index $2 n$ induced new pearly trajectories in the one-point blow up. That is, the only possibility to for $\mathrm{QH}_{*}(\widetilde{L})$ to be distinct from $\mathrm{QH}_{*}(L)$, or at least to have new pearly trajectories on the blow up is if all points in complement of $L \subset(M, \omega)$ are look-alike from the Floer homology perspective.

This naturally leads to the concept of uniruled introduced by P. Biran and $\mathrm{O}$. Cornea [4, Definition 1.1.2]. The pair $(M, L)$ is said to be $(p, q)$-uniruled of order $k$ if for any $p$ distinct points $p_{j} \in M \backslash L$ and any $q$ distinct points $q_{j} \in L$, there exists a subset $\mathcal{J}_{\text {reg }} \subset \mathcal{J}(M, \omega)$ of second category such that for each $J \in \mathcal{J}_{\text {reg }}$ there exists a $J$-holomorphic map $u:(D, \partial D) \rightarrow(M, L)$ such that $p_{j} \in u(D), q_{j} \in u(\partial D)$ and $\mu_{L}([u]) \leq k$. Although the uniruled condition is very restrictive, it is the class of examples where the LFH on the the blow up can differ from the LFH on the base manifold.

The next result summarise how the pearly differential changes in the pearl complex blow up manifold compared with the differential of the pearl complex in the base manifold. For simplicity of the statement we state the result for four-manifolds.

Theorem 1.4. Let $L$ be an admissible Lagrangian in $(M, \omega)$ that is $(1,2)$-uniruled of order $2, x_{0} \in M \backslash L$ a generic point and suppose that $\operatorname{dim}(M)=4$. If $p$ and $q$ in $L$ are critical points of a generic Morse-Smale function $f$ with respect to a generic Riemannian metric $g$ on $L$ such that $\operatorname{ind}(p)-\operatorname{ind}(q)-1=-2$, then

$$
\langle\widetilde{d}(p), q\rangle=\langle d(p), q\rangle+\mathbb{Z}_{2} k
$$

where $k$ is the number, mod 2 , of classes $A \in \mathrm{H}_{2}(M, L ; \mathbb{Z})$ such that for some $J \in$ $\mathcal{J}_{\text {reg }}(M, L)$, such that $\iota^{*} J=J_{0}$ where $\iota$ is the symplectic embedding of the ball, the moduli space of pearly trajectories $\mathcal{P}(p, q, A ; g, f, J)$ is non empty, $\mu_{L}(A)=4$ and there is a J-holomorphic disk $u$ such that $x_{0} \in u(D)$ and $A=[u]$.

Our technique gives an alternative proof of the fact that the Lagrangian induced by the Clifford torus in the one-point blow up of $\left(\mathbb{C} P^{2}, \omega_{\mathrm{FS}}\right)$ is wide. Since the induced Lagrangian torus on the one-point blow of $\left(\mathbb{C} P^{n}, \omega_{\mathrm{FS}}\right)$ is a toric fiber, this fact follows from the theory of K. Fukaya, Y.-G. Oh, H. Ohta and K. Ono of [11]. It was also proved by M. Entov and L. Polterovich in [6]. Recall from [4, Cor. 1.2.12] that the Clifford torus $\mathbb{T}_{\text {Cliff }} \subset\left(\mathbb{C} P^{2}, \omega_{\mathrm{FS}}\right)$ is $(1,1)$-uniruled of order 4 . The prof of the next result is given in Section 6 .

Theorem 1.5. After blowing up one point the proper transform of the Clifford torus $\mathbb{T}_{\text {Cliff }} \subset\left(\mathbb{C} P^{2}, \omega_{\mathrm{FS}}\right)$ in $\left(\widetilde{\mathbb{C} P}{ }^{2}, \widetilde{\omega}_{\rho}\right)$ is also a wide Lagrangian. 
It is also interesting to study the same problem with the possibility of varying the weight of the blow up. However in order to do this, it will be required to use the theory of Kuranishi structures of K. Fukaya, Y.-G. Oh, H. Ohta and K. Ono [9, 10] for LFH and to consider unobstructed Lagrangian submanifolds. No attempt to understand such phenomenon is presented here.

\section{Review OF $\mathrm{HF}_{*}\left(L_{0}, L_{1}\right)$ AND $\mathrm{QH}_{*}(L)$}

2.1. Lagrangian Floer Homology. Throughout this note $(M, \omega)$ will denote a symplectic manifold that is either closed or convex at infinity, $J=\left\{J_{t}\right\}_{0 \leq t \leq 1}$ a family of $\omega$-compatible almost complex structures, and $L_{0}$ and $L_{1}$ compact connected Lagrangian submanifolds that intersect transversally. Let $\mathcal{X}\left(L_{0}, L_{1}\right)$ denote the set of intersection points. Then for $p$ and $q$ in $\mathcal{X}\left(L_{0}, L_{1}\right)$ and $\beta \in \pi_{2}\left(M, L_{0} \cup L_{1}\right)$ denote by $\widehat{\mathcal{M}}(p, q, \beta, J)$ the set of smooth maps $u: \mathbb{R} \times[0,1] \rightarrow M$ such that:

- satisfy the boundary conditions

$$
u(s, 0) \in L_{0} \text {, and } u(s, 1) \in L_{1}, \text { for all } s \in \mathbb{R}
$$

and

$$
\lim _{s \rightarrow-\infty} u(s, t)=q \text { and } \lim _{s \rightarrow+\infty} u(s, t)=p ;
$$

- represent the class $\beta,[u]=\beta$ and

- are $J$-holomorphic,

$$
\bar{\partial}_{J}(u):=\frac{\partial}{\partial s} u(s, t)+J_{t} \frac{\partial}{\partial t} u(s, t)=0 .
$$

The moduli space $\widehat{\mathcal{M}}(p, q, \beta, J)$ admits an action of $\mathbb{R}$, given by $r . u(s, t)=u(s-r, t)$. Denote by $\mathcal{M}(p, q, \beta, J)$ the quotient space of the action. Elements of $\mathcal{M}(p, q, \beta, J)$ are called holomorphic strips; they also received the name of holomorphic disks since $\mathbb{R} \times$ $[0,1] i$ is conformally equivalent to the closed disk minus two points on the boundary.

In some cases the space $\widehat{\mathcal{M}}(p, q, \beta, J)$ is in fact a smooth manifold. To that end, take into account the linearised operator $D_{\bar{\partial}, u}$ of $\bar{\partial}_{J}$ at $u \in \widehat{\mathcal{M}}(p, q, \beta, J)$. Then for integers $k$ and $p$ such that $p>2$ and $k>p / 2$ we have the Sobolev space of vector fields whose $k$-weak derivatives exist and lie in $L_{p}$, and with boundary restrictions;

$$
W_{k}^{p}\left(u^{*} T M ; L_{0}, L_{1}\right):=\left\{\xi \in W_{k}^{p}\left(u^{*} T M\right) \mid \xi(s, 0) \in T L_{0}, \xi(s, 1) \in T L_{1} \text { for all } s \in \mathbb{R}\right\} .
$$

There exists $\mathcal{J}_{\text {reg }}\left(L_{0}, L_{1}\right)$, a dense subset of $\omega$-compatible almost complex structures in $\mathcal{J}\left(L_{0}, L_{1}\right)$ such that for $J \in \mathcal{J}_{\text {reg }}\left(L_{0}, L_{1}\right)$ the linearised operator

$$
D_{\bar{\partial}(J), u}: W_{k}^{p}\left(u^{*} T M ; L_{0}, L_{1}\right) \rightarrow L_{p}\left(u^{*} T M\right) .
$$

is Fredholm and surjective for all $u \in \widehat{\mathcal{M}}(p, q, \beta, J)$. Elements of $\mathcal{J}_{\text {reg }}\left(L_{0}, L_{1}\right)$ are called regular. In this case the index of $D_{\bar{\partial}(J), u}$ equals the Maslov index $\mu_{L_{0}, L_{1}}([u])$ of the homotopy type of $u$ in $\pi_{2}\left(M, L_{0} \cup L_{1}\right)$. Note that in the case when $J$ is regular 
the dimension of the moduli space $\widehat{\mathcal{M}}(p, q, \beta, J)$ is independent of the regular almost complex structure.

Let $\mathrm{CF}\left(L_{0}, L_{1}\right)$ denote the $\Lambda$-vector space generated by the intersection points $\mathcal{X}\left(L_{0}, L_{1}\right)$. Here $\Lambda$ stands for the Novikov field

$$
\Lambda:=\left\{\sum_{j=0}^{\infty} a_{j} T_{j}^{\lambda} \mid a_{j} \in \mathbb{Z}_{2}, \lambda_{j} \in \mathbb{R}, \lim _{j \rightarrow \infty} \lambda_{j}=\infty\right\} .
$$

In the case when $[u] \in \mathcal{M}(p, q, \beta, J), \mu_{L_{0}, L_{1}}([u])=1$ and $J$ is regular the moduli space $\mathcal{M}(p, q, \beta, J)$ is 0 -dimensional and compact, thereby a finite set of points. Set $\#_{\mathbb{Z}_{2}} \mathcal{M}(p, q, \beta, J)$ to be the module 2 number of points of $\mathcal{M}(p, q, \beta, J)$. The Floer differential $\partial_{J}: \mathrm{CF}\left(L_{0}, L_{1}\right) \rightarrow \mathrm{CF}\left(L_{0}, L_{1}\right)$ is defined as

$$
\partial_{J}(p):=\sum_{\substack{q \in \mathcal{X}\left(L_{0} \cap L_{1}\right) \\[u]: \operatorname{index}([u])=1}} \#_{\mathbb{Z}_{2}} \mathcal{M}(p, q,[u], J) T^{\omega([u])} q .
$$

If the Lagrangian submanifolds $L_{0}$ and $L_{1}$ are monotone and the minimal Maslov number of $L_{0}$ and $L_{1}$ is greater than or equal to three, then $\partial_{J} \circ \partial_{J}=0$. In this case the Lagrangian Floer homology of $\left(L_{0}, L_{1}\right)$ is defined as

$$
\operatorname{HF}\left(L_{0}, L_{1}\right):=\frac{\operatorname{ker} \partial_{J}}{\operatorname{im} \partial_{J}} .
$$

Is important to note that the homology group $\operatorname{HF}\left(L_{0}, L_{1}\right)$ does not depend on the regular $\omega$-compatible almost complex structure. When $L_{0}$ and $L_{1}$ are Hamiltonian isotopic and the minimal Maslov number is two, the differential also squares to zero and the Lagrangian Floer homology is also defined in this case.

The role played by the coefficient field $\Lambda$ becomes essential in the definition of the differential $\partial$. In principle the sum in Eq. (2) can be infinite, but by Gromov's compactness there are only finitely many homotopy classes whose energy is below a determined value. Hence $\Lambda$ assures that Eq. (2) is well-defined.

For further details in the definition of Lagrangian Floer homology in the monotone case see [15]; and also [1] and [9] for a more broader class of symplectic manifold where Lagrangian Floer homology is defined.

2.2. Lagrangian quantum homology. The Lagrangian quantum homology is an invariant of a single Lagrangian submanifold. Consider $L \subset(M, \omega)$ a closed monotone Lagrangian submanifold of minimal Maslov number greater than or equal to two. Further, fix a Riemannian metric $g$ on $L$ and Morse-Smale function $f: L \rightarrow \mathbb{R}$. And on $(M, \omega)$ fix an $\omega$-compatible almost complex structure $J \in \mathcal{J}(M, \omega)$.

In the setting of quantum homology of $L \subset(M, \omega), \Lambda$ stands for $\mathbb{Z}_{2}\left[t, t^{-1}\right]$ where the degree of $t$ is $-N_{L}$. Since from the context it will be clear if we are considering LFH or quantum homology; we make no distinction on the notation of $\Lambda$. 
Consider the $\Lambda$-module generated by the critical points of $f$

$$
\mathcal{C}(g, f, J):=\mathbb{Z}_{2}\langle\operatorname{Crit}(f)\rangle \otimes \Lambda
$$

So far, only information about $L$ has been used. The ambient manifold $(M, \omega)$ enters in the definition of the differential. Let $\mathrm{H}_{2}^{D}(M, L)$ denote the image of the Hurewicz homomorphism $\pi_{2}(M, L) \rightarrow \mathrm{H}_{2}(M, L)$. Fix $x, y \in \operatorname{Crit}(f)$ and $A \in \mathrm{H}_{2}^{D}(M, L)$ with $A \neq 0$. An $A$-pearly trajectory from $x$ to $y$, denoted by $\left(u_{1}, \ldots, u_{r} ; l_{0}, \ldots l_{r}\right)_{A}$, is by definition a collection of

- $u_{j}:(D, \partial D) \rightarrow(M, L)$ non constant $J$-holomorphic disks for $j \in\{0, \ldots, r\}$,

- $l_{0}:\left(-\infty, b_{0}\right] \rightarrow L$ and $l_{r}:\left[a_{r}, \infty\right) \rightarrow L$ flow rays of $-\nabla f$,

- $l_{j}:\left[a_{j}, b_{j}\right] \rightarrow L$ flow cords of $-\nabla f$ for $j \in\{1, \ldots, r-1\}$.

These objects are related by the following conditions:

- $A=\left[u_{1}\right]+\cdots+\left[u_{r}\right]$,

- $x=\lim _{t \rightarrow-\infty} l_{0}(t)$,

- $y=\lim _{t \rightarrow \infty} l_{r}(t)$,

- $l_{j}\left(b_{j}\right)=u_{j-1}(-1)$ for $j \in\{0, \ldots, r-1\}$, and

- $l_{j}\left(a_{j}\right)=u_{j}(1)$ for $j \in\{1, \ldots, r\}$.

Two $A$-pearly trajectories $\left(u_{1}, \ldots, u_{r} ; l_{0}, \ldots l_{r}\right)_{A}$ and $\left(u_{1}^{\prime}, \ldots, u_{s}^{\prime} ; l_{0}^{\prime}, \ldots l_{s}^{\prime}\right)_{A}$ are said to be equivalent if $r=s$ and for every $j \in\{1, \ldots, r\}$ there exists $\sigma_{j} \in \operatorname{Aut}(D)$ such that $\sigma_{j}(-1)=-1, \sigma_{j}(1)=1$ and $u_{j}^{\prime}=u_{j} \circ \sigma_{j}$. The collection of all equivalence classes of $A$-pearly trajectories is denoted by $\mathcal{P}(x, y, A ; g, f, J)$. In the case when $A=0$, by definition, the space $\mathcal{P}(x, y, 0 ; g, f, J)$ consists of unparameterised flow lines of $-\nabla f$ that connect $x$ to $y$. That is, Morse trayectories.

The space $\mathcal{P}(x, y, A ; g, f, J)$ is a smooth manifold of dimension

$$
\delta(x, y, A):=\operatorname{ind}_{f}(x)-\operatorname{ind}_{f}(y)-1+\mu(A) .
$$

where $\operatorname{ind}_{f}(\cdot)$ stands for the Morse index with respect to $f$ of the critical point. Recall that the Riemannian metric $g$ and $f: L \rightarrow \mathbb{R}$ are subject to the condition that $f$ is a Morse-Samale function. If follows from [3, Prop. 3.2], see also [4], that there is dense subset $\mathcal{J}_{\text {reg }}(M, L)$ in the space of $\omega$-compatible almost complex structures $\mathcal{J}(M, \omega)$ such that for $J \in \mathcal{J}_{\text {reg }}(M, L)$ if $\delta(x, y, A)=0$ then $\mathcal{P}(x, y, A ; g, f, J)$ is a finite set.

Fix $J \in \mathcal{J}_{\text {reg }}(M, L)$. Then for $x \in \operatorname{Crit}(f)$ the differential is defined as

$$
d_{J}(x):=\sum_{y \in \operatorname{Crit}(f),} \#_{A \in \mathrm{H}_{2}^{D}(M, L)} \mathcal{\mathbb { Z } _ { 2 }} \mathcal{P}(x, y, A ; g, f, J) T^{\mu(A) / N_{L}} y
$$

where the sum is over all $y$ and $A$ such that $\delta(x, y, a)=0$. Further, $d_{J}$ extends $\Lambda$ linearly to $\mathcal{C}(g, f, J)$. $\left(\mathcal{C}(g, f, J), d_{J}\right)$ is called the pearl complex of $(M, L, g, f, J)$. It's homology is called the Lagrangian quantum homology of $L \subset(M, \omega)$

$$
\mathrm{QH}_{*}(L):=\mathrm{H}_{*}\left(\mathcal{C}(g, f, J), d_{J}\right)=\frac{\operatorname{ker} d_{J}}{\operatorname{im} d_{J}} .
$$


Further details about the definition of the $\Lambda$-module $\mathrm{QH}_{*}(L)$, as well as the invariance of the Riemannian metric $g$, the Morse-Smale function $f$ and $J \in \mathcal{J}_{\text {reg }}(M, L)$, can be found in [3, 4].

\section{REVIEW OF THE SYMPLECTIC ONE-POINT BLOW UP}

The symplectic one-point blow up plays a fundamental role in this note. Hence we review the definitions of the complex and symplectic one-point blow up. To that end, consider the complex blow up of $\mathbb{C}^{n}$ at the origin $\Phi: \widetilde{\mathbb{C}^{n}} \rightarrow \mathbb{C}^{n}$, where $n>1$. That is

$$
\widetilde{\mathbb{C}^{n}}=\left\{(z, \ell) \in \mathbb{C}^{n} \times \mathbb{C} P^{n-1} \mid z \in \ell\right\}
$$

and the blow up map is given by $\Phi(z, \ell)=z$. For $r>0$, let $L(r):=\Phi^{-1}\left(\operatorname{int}\left(B^{2 n}(r)\right)\right)$ where $B^{2 n}(r) \subset \mathbb{C}^{n}$ is the closed ball and $\operatorname{int}(\cdot)$ stands for the interior of the set.

If $(M, J)$ is a complex manifold and $\iota:\left(\operatorname{int} B^{2 n}(r), J_{0}\right) \rightarrow(M, J)$ is such that $\iota^{*} J=J_{0}$ and $x_{0}=\iota(0)$, then the complex blow up of $M$ at $x_{0}$ is defined as

$$
\widetilde{M}:=\left(M \backslash\left\{x_{0}\right\}\right) \cup L(r) / \sim
$$

where $z=\iota\left(z^{\prime}\right) \in \iota\left(\operatorname{int}\left(B^{2 n}(r)\right)\right) \backslash\left\{x_{0}\right\}$ is identified with the unique point $\left(z^{\prime}, \ell_{z^{\prime}}\right) \in$ $L(r)$ and $\ell_{z^{\prime}}$ is the line determined by $z^{\prime}$. So defined, $\widetilde{M}$ carries a unique complex structure $\widetilde{J}$ such that the blow up map $\pi:(\widetilde{M}, \widetilde{J}) \rightarrow(M, J)$ is $(\widetilde{J}, J)$-holomorphic. The preimage of the blown up point $\pi^{-1}\left(x_{0}\right)=E$ is called the exceptional divisor. Further, the blow up map induces a biholomorphic map $\widetilde{M} \backslash E \rightarrow M \backslash\left\{x_{0}\right\}$.

The next task is to define the symplectic one-point blow up. The symplectic blow up relies on the complex blow up. However there is not a unique symplectic blow up; there is a whole family of symplectic forms on the one-point blow up.

As a first step we look at $\left(\mathbb{C}^{n}, \omega_{0}\right)$ and define a symplectic structure on $\widetilde{\mathbb{C}^{n}}$. Here $\omega_{0}$ is the standard symplectic form on euclidean space. For $\rho>0$, consider the symplectic form

$$
\omega(\rho):=\Phi^{*}\left(\omega_{0}\right)+\rho^{2} p r^{*}\left(\omega_{\mathrm{FS}}\right)
$$

on $\widetilde{\mathbb{C}^{n}}$ where $p r: \widetilde{\mathbb{C}^{n}} \rightarrow \mathbb{C} P^{n-1}$ is the canonical line bundle and the Fubini-Study form $\left(\mathbb{C} P^{n-1}, \omega_{\mathrm{FS}}\right)$ is normalised so that the area of every line is $\pi$. Note that on the exceptional divisor the symplectic form $\omega(\rho)$ restricts to $\rho^{2} \omega_{\mathrm{FS}}$. Hence in $\left(\widetilde{\mathbb{C}^{n}}, \omega(\rho)\right)$ the area of any line in the exceptional divisor is $\rho^{2} \pi$.

Next, the symplectic form $\omega(\rho)$ is perturb in such a way so that in the complement of a neighbourhood of the exceptional divisor agrees with the standard symplectic form $\omega_{0}$. Once this is done, following the definition of the blow up manifold (3) it will be possible to define a symplectic form on $\widetilde{M}$.

For $r>\rho$ let $\beta:[0, r] \rightarrow[\rho, r]$ be any smooth function such that

$$
\beta(s):= \begin{cases}\sqrt{\rho^{2}+s^{2}} & \text { for } 0 \leq s \leq \delta \\ s & \text { for } r-\delta \leq s \leq r .\end{cases}
$$


and on the remaining part takes any value as long as $0<\beta^{\prime}(s) \leq 1$ for $0<s \leq r-\delta$. Then $F_{\rho}: L(r) \backslash E \rightarrow \operatorname{int}\left(B^{2 n}(r)\right) \backslash B^{2 n}(\rho)$ defined as

$$
F_{\rho}(z):=\beta(|z|) \frac{z}{|z|}
$$

is a diffeomorphism such that $\widetilde{\omega}(\rho):=F_{\rho}^{*}\left(\omega_{0}\right)$ is a symplectic form. So defined $\widetilde{\omega}(\rho)$ is such that

- $\widetilde{\omega}(\rho)=\omega_{0}$ on $\operatorname{int}(L(r) \backslash L(r-\delta))$ and

- $\widetilde{\omega}(\rho)=\omega(\rho)$ on $L(\delta)$.

We call $(L(r), \widetilde{\omega}(\rho))$ the local model of the symplectic one-point blow up.

In order to define the symplectic blow up of $(M, \omega)$ at $x_{0}$, it is requiere a symplectic embedding $\iota:\left(B^{2 n}(\rho), \omega_{0}\right) \rightarrow(M, \omega)$ and an almost complex structure $J$ on $(M, \omega)$ such that $\iota(0)=x_{0}$ and $\iota^{*} J=J_{0}$. Notice that the symplectic embedding $\iota$ extends to $\operatorname{int}\left(B^{2 n}(r)\right)$ for $r$ such that $r-\rho$ is small.

Finally, using the symplectic embedding as a symplectic chart and the local model $(L(r), \widetilde{\omega}(\rho))$ defined above, the symplectic form of weight $\rho$ on $\widetilde{M}$ is defined as

$$
\widetilde{\omega}_{\rho}:= \begin{cases}\omega & \text { on } \pi^{-1}\left(M \backslash \iota B^{2 n}\left(\sqrt{\rho^{2}+\delta^{2}}\right)\right) \\ \widetilde{\omega}(\rho) & \text { on } L_{r} .\end{cases}
$$

For further details and the dependence of the symplectic blow up on the choices that we made see [12], [13] and [14]. The above observations are summarised in the next proposition.

Proposition 3.1. Let $(M, \omega)$ be a symplectic manifold, $\iota:\left(B^{2 n}(r), \omega_{0}\right) \rightarrow(M, \omega)$ a symplectic embedding and $J$ a $\omega$-compatible almost complex structure such that $\iota(0)=x_{0}$ and $\iota^{*} J=J_{0}$. If $\rho<r$, then the symplectic blow up $\pi:\left(\widetilde{M}, \widetilde{\omega}_{\rho}\right) \rightarrow(M, \omega)$ of weight $\rho$ satisfies:

(1) $\pi: \widetilde{M} \backslash E \rightarrow M \backslash\left\{x_{0}\right\}$ is a diffeomorphism,

(2) $\pi^{*}(\omega)=\widetilde{\omega}_{\rho}$ on $\pi^{-1}\left(M \backslash \iota B^{2 n}(r)\right)$, and

(3) the area of the line in $E$ is $\rho^{2} \pi$.

(4) $\widetilde{J}$ is $\widetilde{\omega}_{\rho}$-compatible.

From now on assume that $J$ on $(M, \omega)$ satisfies the condition $\iota^{*} J=J_{0}$, where $J_{0}$ is the standard complex structure on $\mathbb{C}^{n}$. It is well known that this condition induces a unique almost complex structure $\widetilde{J}$ on $\left(\widetilde{M}, \widetilde{\omega}_{\rho}\right)$ such that the blow up map $\pi:\left(\widetilde{M}, \widetilde{\omega}_{\rho}\right) \rightarrow(M, \omega)$ is $(J, \widetilde{J})$-holomorphic. In particular $\pi: \widetilde{M} \backslash E \rightarrow M \backslash\left\{x_{0}\right\}$ is a biholomorphic map.

However note that is possible that the set $\mathcal{J}_{\iota}:=\left\{J \in \mathcal{J}(M, \omega) \mid \iota^{*} J=J_{0}\right\}$ might not contain a regular almost complex structure. For the map $\iota^{*}: \mathcal{J}(M, \omega) \rightarrow$ $\mathcal{J}\left(\iota\left(B^{2 n}(\rho)\right), \omega\right)$ that restricts an almost complex structure to the image of the embedded ball is continuous. Hence $\mathcal{J}_{\iota}=\left(\iota^{*}\right)^{-1}\left(J_{0}\right)$ is closed. 
Lemma 3.2. Let $\iota:\left(B^{2 n}(r), \omega_{0}\right) \rightarrow(M, \omega)$ be a symplectic embedding and $\mathcal{J}_{\iota}:=$ $\left\{J \in \mathcal{J}(M, \omega) \mid \iota^{*} J=J_{0}\right\}$. Then $\mathcal{J}_{\iota} \subset \mathcal{J}(M, \omega)$ is closed and its complement is dense

\section{LAGRANGIAN SUBMANIFOLDS AND HOLOMORPHIC DISKS}

4.1. Lift of holomorphic disks. Fix a symplectic embedding $\iota:\left(B^{2 n}(\rho), \omega_{0}\right) \rightarrow$ $(M, \omega)$ and set $x_{0}:=\iota(0)$ to be the base point. Consider $L \subset(M, \omega)$ a Lagrangian submanifold such that $L \cap \iota B^{2 n}(\rho)$ is empty. Then by part (2) of Proposition 3.1 it follows that $\widetilde{L}:=\pi^{-1}(L)$ is a Lagrangian submanifold in $\left(\widetilde{M}, \widetilde{\omega}_{\rho}\right)$. However if $L$ is such that $L \cap \iota B^{2 n}(\rho)$ is not empty, then $\widetilde{L}$ is not necessarily a Lagrangian submanifold, even if $x_{0} \notin L$.

Let $J$ be a $\omega$-compatible almost complex structure on $(M, \omega)$ as in Section 3 and $\widetilde{J}$ the unique $\widetilde{\omega}_{\rho}$-compatible almost complex structure on $\left(\widetilde{M}, \widetilde{\omega}_{\rho}\right)$ such that the blow up map $\pi:(M, \omega) \rightarrow\left(\widetilde{M}, \widetilde{\omega}_{\rho}\right)$ is $(J, \widetilde{J})$-holomorphic. Hence $\pi: \widetilde{M} \backslash E \rightarrow M \backslash\left\{x_{0}\right\}$ is a biholomorphic map, therefore any $\widetilde{J}$-holomorphic disk $\widetilde{u}:(D, \partial D) \rightarrow(\widetilde{M}, \widetilde{L})$ projects to a $J$-holomorphic disk $\pi \circ \widetilde{u}$ on $(M, L)$. And vice versa, if $u$ is a $J$-holomorphic disc on $(M, L)$ such that $x_{0} \notin u(D)$, then $\widetilde{u}:=\pi^{-1} \circ u$ is a $\widetilde{J}$-holomorphic $\operatorname{disc}$ in $(\widetilde{M}, \widetilde{L})$. Recall that we are assuming that the Lagrangian submanifold $L \subset(M, \omega)$ does not contain the base point $x_{0}$.

It only remains to analyse the lift of the $J$-holomorphic disk $u:(D, \partial D) \rightarrow(M, L)$ in the case when $x_{0} \in u(D)$. Since the base point $x_{0}$ is not on the Lagrangian submanifold $L \subset(M, \omega)$ and the almost complex structure satisfies $\iota^{*} J=J_{0}$, in order to define the lift of $u$ to $\left(\widetilde{M}, \widetilde{\omega}_{\rho}\right)$ we ignore the submanifold $L$ and consider the case $(M, \omega)=\left(\mathbb{C}^{n}, \omega_{0}\right)$ blown up at the the origin. Thus $x_{0}=0$ and $u: D \rightarrow \mathbb{C}^{n}$ is holomorphic with respect to the standard complex structure and $0 \in u(D)$.

For let $u: D \rightarrow \mathbb{C}^{n}$ be a non constant holomorphic map such that $u(0)=0$. Then each non constant component function $u_{j}: D \rightarrow \mathbb{C}$ of $u$ can be written as $u_{j}=z^{k_{j}} h_{j}$ where $k_{j}$ is the order of the zero of $u_{j}$ at $0 \in D$. Thus $k_{j}$ is a positive integer and $h_{j}$ is holomorphic function that does not vanish at 0 . Except if $u_{j} \equiv 0$, in this case we set $k_{j}$ to be equal to $\infty$. Thus the holomorphic map $u$ can be expressed as

$$
u(z)=z^{k}\left(\hat{h}_{1}(z), \ldots, \hat{h}_{n}(z)\right)
$$

where $k:=\min k_{j}$ and at least one coordinate function $\hat{h}_{j}$ does not vanish at 0 since we assumed that $u$ is non constant. The lift $\widetilde{u}: D \rightarrow \widetilde{\mathbb{C}}^{n}$ of $u$ is defined as

$$
\widetilde{u}(z):=\left(u(z),\left[\hat{h}_{1}(z): \cdots: \hat{h}_{n}(z)\right]\right) .
$$

So defined, $\widetilde{u}$ is holomorphic and projects to $u$ under the blow up map. Notice that if $\widetilde{u}_{0}$ is another $\widetilde{J}$-holomorphic lift of $u$, then $\widetilde{u}$ and $\widetilde{u}_{0}$ agree on $D \backslash \widetilde{u}^{-1}(E)$. Since the maps are $\widetilde{J}$-holomorphic they agree on all $D$, thus the holomorphic lift of $u$ is unique. 
Remark. If $\psi: \mathbb{C}^{n} \rightarrow \mathbb{C}^{n}$ is a biholomorphic map such that $\psi(0)=0$ and $u: D \rightarrow \mathbb{C}^{n}$ is as above then the factorisation of $\psi \circ u$ as in Eq. (4) gives the same value of $k$ as that of $u$. Thus $k$ is independent of the coordinate system.

Now that we have defined the lift of $u:(D, \partial D) \rightarrow(M, L)$ to $\left(\widetilde{M}, \widetilde{\omega}_{\rho}\right)$, there is one more consideration that needs attention; the behaviour of $u$ at the blown up point $x_{0}$. If $u:(D, \partial D) \rightarrow(M, L)$ is a non constant $J$-holomorphic disk and $z \in D \backslash \partial D$ is such that $u(z)=x_{0}$, then we define the multiplicity of $u$ at $z$ has the integer $k \in\{0,1, \ldots, \infty\}$ that appears in Eq. (4). Also we define the multiplicity of $u$ at $x_{0}$ as $k_{1}+\cdots+k_{r}$ where $u^{-1}\left(x_{0}\right)=\left\{z_{1}, \ldots, z_{r}\right\}$ and the multiplicity of $u$ at $z_{j}$ is $k_{j}$. In the case when $x_{0}$ is not in the image of $u$, we say that $u$ has multiplicity zero at $x_{0}$. Recall that since $u$ is $J$-holomorphic, the preimage of a point under $u$ is a finite set.

Proposition 4.1. Let $J$ be an almost complex structure on $(M, \omega)$ as above, such that $\iota^{*} J=J_{0}$ and $\widetilde{J}$ the unique almost complex structure on $\left(\widetilde{M}, \widetilde{\omega}_{\rho}\right)$ such that $\pi$ is $(\widetilde{J}, J)$-holomorphic. If $u:(D, \partial D) \rightarrow(M, L)$ a non constant $J$-holomorphic disk, then there exists a unique $\widetilde{J}$-holomorphic map $\widetilde{u}:(D, \partial D) \rightarrow(\widetilde{M}, \widetilde{L})$ such that $\pi \circ \widetilde{u}=u$. Moreover if $u$ has multiplicity $k$ at $x_{0}$, then $\widetilde{u} \cdot E=k$.

Proof. It only remains to prove the relation $\widetilde{u} \cdot E=k$. Since the underlying manifold $\left(\widetilde{M}, \widetilde{\omega}_{\rho}\right)$ agrees with the complex blow up and the exceptional divisor $E$ is a $\widetilde{J}$-holomorphic submanifold of real codimension two of $\left(\widetilde{M}, \widetilde{\omega}_{\rho}\right)$. Now the multiplicity of $u$ at $x_{0}$ is $k$, therefore $\widetilde{u} \cdot E=k$.

Recall from Theorem 1.2 the invariance of the blown up point. Thus, the holomorphic disks that will by considered in the framework of LFH would have $k=0$, that it the case when the blown up point is irrelevant for the LFH; or $k=1$ that appears in the presence of some form of uniruled condition.

4.2. Monotone Lagrangians on blow ups. For a Lagrangian submanifold $L$ of $(M, \omega)$, there exist two classical morphisms

$$
I_{\mu, L}: \pi_{2}(M, L) \rightarrow \mathbb{Z} \quad \text { and } \quad I_{\omega, L}: \pi_{2}(M, L) \rightarrow \mathbb{R}
$$

the Maslov index and symplectic area morphisms respectively. A Lagrangian submanifold is said to be monotone if there exists $\lambda>0$ such that $I_{\mu, L}=\lambda \cdot I_{\omega, L}$. The constant $\lambda$ is called the monotonicity constant of $L$. As mentioned in Section 2, in order to define Lagrangian Floer homology one restricts to monotone Lagrangians. Thus if $L \subset(M, \omega)$ is a monotone Lagrangian submanifold we need to guarantee that $\widetilde{L}$ is a monotone Lagrangian on the one-point blow up $\left(\widetilde{M}, \widetilde{\omega}_{\rho}\right)$.

If $L$ is a monotone Lagrangian submanifold of $(M, \omega)$ with monotonicity constant $\lambda$, then $(M, \omega)$ is monotone symplectic. That is $I_{c}=(\lambda / 2) I_{\omega}$. In this case $I_{\omega}$ is defined on $\pi_{2}(M)$ and the morphism $I_{c}: \pi_{2}(M) \rightarrow \mathbb{Z}$ is given by evaluating at the first Chern 
class of $(M, \omega)$ with respect to any almost complex structure. Here $\alpha:=\lambda / 2$ is the monotonicity constant of $(M, \omega)$.

The first Chern classes of $(M, \omega)$ and the one-point blow $\left(\widetilde{M}, \widetilde{\omega}_{\rho}\right)$ are related by the equation

$$
c_{1}(\widetilde{M})=\pi^{*}\left(c_{1}(M)\right)-(n-1) \mathrm{PD}_{\widetilde{M}}(E)
$$

where $E$ is the class of the exceptional divisor. Recall that if $L_{E}$ stands for the complex line in the exceptional divisor in $\left(\widetilde{M}, \widetilde{\omega}_{\rho}\right)$, then $\widetilde{\omega}_{\rho}\left(L_{E}\right)=\pi \rho^{2}$ and $\operatorname{PD}_{\widetilde{M}}(E)\left(\left[L_{E}\right]\right)=$ -1 . Since the underlying manifold for the symplectic blow up is independent of the weight it follows from Eq. (6) the symplectic one-point blow up $\left(\widetilde{M}, \widetilde{\omega}_{\rho}\right)$ is monotone if and only if

$$
\rho^{2}=\frac{n-1}{\alpha \pi}=\frac{2(n-1)}{\lambda \pi} .
$$

Furthermore if Eq. (7) holds, then $\left(\widetilde{M}, \widetilde{\omega}_{\rho}\right)$ and $(M, \omega)$ have the same monotonicity constant.

Throughout the paper we make the following assumptions. If the condition of monotonicity on $(M, \omega)$ is required, we will assume that the Gromov's width of $(M, \omega)$ is greater than $n-1 / \alpha$, where $\alpha$ is its monotonicity constant; and the weight $\rho$ of the one-point blow up $\left(\widetilde{M}, \widetilde{\omega}_{\rho}\right)$ is subject to Eq. (77).

The two homotopy long exact sequences of the pairs $(\widetilde{M}, \widetilde{L})$ and $(M, L)$ are related by the blow up map, in the sense that the diagram

$$
\begin{aligned}
& \rightarrow \pi_{2}(\widetilde{L}) \stackrel{\widetilde{i}_{*}}{\rightarrow} \pi_{2}(\widetilde{M}) \stackrel{\tilde{j}_{*}}{\rightarrow} \pi_{2}(\widetilde{M}, \widetilde{L}) \stackrel{\widetilde{\delta}}{\rightarrow} \pi_{1}(\widetilde{L}) \rightarrow \pi_{1}(\widetilde{M}) \rightarrow \\
& \downarrow=\quad \downarrow \pi_{*} \quad \downarrow \pi_{*} \quad \downarrow=\quad \simeq \downarrow \pi_{*} \\
& \rightarrow \pi_{2}(L) \stackrel{i_{*}}{\rightarrow} \pi_{2}(M) \stackrel{j_{*}}{\rightarrow} \pi_{2}(M, L) \stackrel{\delta}{\rightarrow} \pi_{1}(L) \rightarrow \pi_{1}(M) \rightarrow
\end{aligned}
$$

is commutative.

Lemma 4.2. If $L$ is a Lagrangian submanifold in $(M, \omega)$ then the $\operatorname{map} \pi_{*}: \pi_{2}(\widetilde{M}, \widetilde{L}) \rightarrow$ $\pi_{2}(M, L)$ is surjective and

$$
\operatorname{ker}\left\{\pi_{*}: \pi_{2}(\widetilde{M}, \widetilde{L}) \rightarrow \pi_{2}(M, L)\right\}=\widetilde{j}_{*} \operatorname{ker}\left\{\pi_{*}: \pi_{2}(\widetilde{M}) \rightarrow \pi_{2}(M)\right\}
$$

Proof. As smooth manifolds $\widetilde{M} \simeq M \# \mathbb{C} P^{n}$. Therefore $\pi_{*}: \pi_{2}(\widetilde{M}) \rightarrow \pi_{2}(M)$ is surjective. Hence four of the vertical maps in the above diagram are surjective, hence so is the map in the middle $\pi_{*}: \pi_{2}(\widetilde{M}, \widetilde{L}) \rightarrow \pi_{2}(M, L)$.

Now we show that the kernel of $\pi_{*}: \pi_{2}(\widetilde{M}, \widetilde{L}) \rightarrow \pi_{2}(M, L)$ is contained in $\widetilde{j}_{*}$ ker $\left\{\pi_{*}\right.$ : $\left.\pi_{2}(\widetilde{M}) \rightarrow \pi_{2}(M)\right\}$; the reverse inclusion follows by the commutativity of the diagram.

For, let $u \in \pi_{2}(\widetilde{M}, \widetilde{L})$ be an element that maps to the identity element $e \in \pi_{2}(M, L)$. Since $\pi_{1}(L)=\pi_{1}(\widetilde{L})$ then $\widetilde{\delta}(u)=e$ and by exactness of the top sequence there is $w \in \pi_{2}(\widetilde{M})$ such that $\widetilde{j}_{*}(w)=u$. Note that $\pi_{*}\left(\widetilde{j}_{*}(w)\right)=e$. Thus by exactness and the 
fact that $\pi_{2}(L)=\pi_{2}(\widetilde{L})$, there is $w^{\prime} \in \pi_{2}(\widetilde{L})$ such that $\pi_{*}\left(\widetilde{i}_{*}\left(w^{\prime}\right)\right)=\pi_{*}(w)$. Therefore $\widetilde{j}_{*}\left(w-\widetilde{i}_{*}\left(w^{\prime}\right)\right)=u$ and $w-\widetilde{i}_{*}\left(w^{\prime}\right)$ maps to $e \in \pi_{2}(M)$.

If follows from Lemma 4.2 that elements of the kernel of $\pi_{*}: \pi_{2}(\widetilde{M}, \widetilde{L}) \rightarrow \pi_{2}(M, L)$ are induced by absolute classes. Therefore any $[\widetilde{u}] \in \pi_{2}(\widetilde{M}, \widetilde{L})$ can be expressed as $[\widetilde{u}]=\left[u_{0} \# w\right]$ where $[w] \in \operatorname{im}\left\{\pi_{2}(\widetilde{M}) \rightarrow \pi_{2}(\widetilde{M}, \widetilde{L})\right\}$ and $u_{0}:(D, \partial D) \rightarrow(\widetilde{M}, \widetilde{L})$ does not intersect the exceptional divisor.

Lemma 4.3. If $L$ is a Lagrangian submanifold in $(M, \omega)$ and $[u] \in \pi_{2}(\widetilde{M}, \widetilde{L})$, then there exist $\ell \in \mathbb{Z}$ and $u_{0}:(D, \partial D) \rightarrow(\widetilde{M}, \widetilde{L})$ such that $u_{0}$ does not intersect the exceptional divisor and

$$
[u]=\left[u_{0} \# \widetilde{j}\left(\ell\left[L_{E}\right]\right)\right] .
$$

Proof. First assume that $[u]$ is such that $\pi_{*}[u]=e$. Then by Lemma 4.2 there exists $\left[w_{0}\right] \in \pi_{2}(\widetilde{M})$ such that $[u]=\widetilde{j}_{*}\left[w_{0}\right]=\left[\widetilde{j} \circ w_{0}\right]$. Let $[v]:=\pi_{*}\left[w_{0}\right]$, thus $j_{*}[v]=e$. Then by the commutativity of the above diagram and the fact that $\pi_{2}(\widetilde{L})=\pi_{2}(L)$, there is $\left[v_{0}\right] \in \pi_{2}(\widetilde{L})$ such that $\pi_{*} \circ \widetilde{i}_{*}\left[v_{0}\right]=[v]$. Therefore $\left[w_{0}\right]-\widetilde{i}_{*}\left[v_{0}\right] \in \pi_{2}(\widetilde{M})$ is such that $\widetilde{j}_{*}\left(\left[w_{0}\right]-\widetilde{i}_{*}\left[v_{0}\right]\right)=[u]$ and maps to $e$ under $\pi_{*}$. Thus the result holds in this case.

Now for arbitrary $[u] \in \pi_{2}(\widetilde{M}, \widetilde{L})$, let $\left[u_{0}\right]:=\pi_{*}[u] \in \pi_{2}(M, L)$. Since $x_{0}$ is not in $L$, there exist a continuous map $u_{0}^{\prime}:(D, \partial D) \rightarrow(M, L)$ such that $x_{0}$ is not in $u_{0}^{\prime}(D)$ and $\left[u_{0}\right]=\left[u_{0}^{\prime}\right]$. In particular, the map $u_{0}^{\prime}$ lifts to a map $(\widetilde{M}, \widetilde{L})$ that does not intersect the exceptional divisor. Let $\widetilde{u}_{0}$ be such a map, thus $\left[\widetilde{u}_{0}\right] \in \pi_{2}(\widetilde{M}, \widetilde{L})$ and $\left[\pi \circ \widetilde{u}_{0}\right]=\left[u_{0}^{\prime}\right]$. Notice that $[u]-\left[\widetilde{u}_{0}\right]$ maps to $e$ under $\pi_{*}$. Hence there exists $[w] \in \pi_{2}(\widetilde{M})$ such that $\pi_{*}[w]=e$ and

$$
[u]=\left[\widetilde{u}_{0}\right]+\widetilde{j}_{*}[w]=\left[\widetilde{u}_{0} \#(\widetilde{j} \circ w)\right] .
$$

Further since $\pi_{*}[w]=e$ then as a homology class $[w]$ is a purely exceptional class. This means that $[w]=\ell\left[L_{E}\right]$ for some $\ell \in \mathbb{Z}$. Therefore $[u]=\left[u_{0} \# \widetilde{j}\left(\ell\left[L_{E}\right]\right)\right]$.

With these results is now possible to show that the lift to the one-point blow up of a monotone Lagrangian submanifold is also monotone.

Lemma 4.4. Let $L$ be a Lagrangian submanifold in $(M, \omega)$. If $\widetilde{u}:(D, \partial D) \rightarrow(\widetilde{M}, \widetilde{L})$ is a smooth map, then

$$
\mu_{\widetilde{L}}[\widetilde{u}]=\mu_{L}[\pi \circ \widetilde{u}]+2(n-1) \ell
$$

for some $\ell \in \mathbb{Z}$.

Next, we see that the condition of monotonicity of a Lagrangian submanifold is preserved by the proper transform.

Lemma 4.5. Let $L$ be a Lagrangian submanifold in $(M, \omega)$, then $\widetilde{L}$ is monotone Lagrangian submanifold of $\left(\widetilde{M}, \widetilde{\omega}_{\rho}\right)$ with the same monotonicity constant as $L$. 
Proof. Let $\lambda$ and $\alpha:=\lambda / 2$ be the monotonicity constants of $L \subset(M, \omega)$ and $(M, \omega)$ respectively. Recall that the value of $\rho=\sqrt{\frac{n-1}{\alpha \pi}}$ is such that $\left(\widetilde{M}, \widetilde{\omega}_{\rho}\right)$ is monotone with monotonicity constant $\alpha$.

For $[\widetilde{u}]$ in $\pi_{2}(\widetilde{M}, \widetilde{L})$, by Lemma 4.3 we have that $[\widetilde{u}]=\left[u_{0} \# \widetilde{j}\left(\ell\left[L_{E}\right]\right)\right]$ where $u_{0}$ : $(D, \partial D) \rightarrow(\widetilde{M}, \widetilde{L})$ does not intersect the exceptional divisor and $\ell \in \mathbb{Z}$. Therefore

$$
I_{\widetilde{\mu}, \widetilde{L}}([\widetilde{u}])=I_{\widetilde{\mu}, \widetilde{L}}\left(\left[u_{0}\right]\right)+2 c_{1}(\widetilde{M})\left(\ell\left[L_{E}\right]\right) .
$$

Since $u_{0}$ does not intersects the exceptional divisor, we can assume that its image lies in $\widetilde{M} \backslash \pi^{-1}\left(\iota B^{2 n}(\rho)\right)$. By Proposition 3.1, $\left(\widetilde{M} \backslash \pi^{-1}\left(\iota B^{2 n}(\rho)\right), \widetilde{\omega}_{\rho}\right)$ is symplectomorphic to $\left(M \backslash \iota B^{2 n}(\rho), \omega\right)$ under the blow up map. The Maslov index is invariant under symplectic diffeomorphisms, thus $I_{\widetilde{\mu}, \widetilde{L}}\left(\left[u_{0}\right]\right)=I_{\mu, L}\left(\left[\pi \circ u_{0}\right]\right)$ and

$$
I_{\widetilde{\mu}, \widetilde{L}}([\widetilde{u}])=I_{\mu, L}\left(\left[\pi \circ u_{0}\right]\right)+2 \ell c_{1}(\widetilde{M})\left(\left[L_{E}\right]\right) .
$$

Recall that $\widetilde{\omega}_{\rho}\left(\left[L_{E}\right]\right)=\pi \rho^{2}$ and

$$
c_{1}(\widetilde{M})\left[L_{E}\right]=\pi^{*}\left(c_{1}(M)\right)\left[L_{E}\right]-(n-1) \mathrm{PD}_{\widetilde{M}}(E)\left[L_{E}\right]=n-1 .
$$

Thus

$$
\begin{aligned}
I_{\mu, L}\left(\left[\pi \circ u_{0}\right]\right)+2 \ell c_{1}(\widetilde{M})\left(\left[L_{E}\right]\right) & =I_{\mu, L}\left(\left[\pi \circ u_{0}\right]\right)+2(n-1) \ell \\
& =\lambda I_{\omega}\left(\left[\pi \circ u_{0}\right]\right)+2(n-1) \ell \\
& =\lambda I_{\omega}\left(\left[\pi \circ u_{0}\right]\right)+(2 \alpha) \pi \rho^{2} \ell \\
& =\lambda \omega\left(\left[\pi \circ u_{0}\right]\right)+\lambda I_{\widetilde{\omega}_{\rho}}([w])=\lambda I_{\widetilde{\omega}_{\rho}}([\widetilde{u}]) .
\end{aligned}
$$

That is, $I_{\widetilde{\mu}, \widetilde{L}}([\widetilde{u}])=\lambda I_{\widetilde{\omega}_{\rho}}([\widetilde{u}])$ for all $[\widetilde{u}] \in \pi_{2}(\widetilde{M}, \widetilde{L})$ and $\widetilde{L}$ is a monotone Lagrangian submanifold.

Notice from the above proof, that the Maslov index of $\widetilde{u}:(D, \partial D) \rightarrow(\widetilde{M}, \widetilde{L})$ can be written in terms of the Maslov index of $\pi \circ \widetilde{u}$. In particular, in the case of a $J$-holomorphic disk, we have a precise description of the integer $\ell$ that appears in the above formula.

Proposition 4.6. Let $(M, \omega)$ and $L$ as in Lemma 4.5 and $J$ a $\omega$-compatible almost complex structure on $(M, \omega)$. If $\widetilde{u}:(D, \partial D) \rightarrow(\widetilde{M}, \widetilde{L})$ is $\widetilde{J}$-holomorphic and $[\widetilde{u}] \cdot E=$ $\ell \geq 0$, then

$$
\mu_{\widetilde{L}}[\widetilde{u}]=\mu_{L}[\pi \circ \widetilde{u}]-2(n-1) \ell .
$$

Hence the holomorphic disks $\widetilde{u}$ and $\pi \circ \widetilde{u}$ have the same Maslov index if and only if $\widetilde{u}$ does not intersect the exceptional divisor, or equivalently $\pi \circ \widetilde{u}$ does not contain the base point $x_{0}$.

Finally recall that the minimal Maslov number $N_{L}$ of a Lagrangian submanifold $L$ in $(M, \omega)$ is defined as the the positive generator of the image of $I_{\mu, L}: \pi_{2}(M, L) \rightarrow \mathbb{Z}$. Then under the considerations of Lemma 4.4, $N_{\widetilde{L}} \leq N_{L}$. 
Remark. The statements presented in this section regarding the Maslov index $\mu_{L}$ of a Lagrangian submanifold $L$, also apply to the relative Maslov index $\mu_{L_{0}, L_{1}}$ of the pair of Lagrangian submanifolds $L_{0}$ and $L_{1}$.

\section{LAgRANGian Floer homology ON THE BLOW UP}

Let $(M, \omega)$ be a symplectic manifold that is either closed or convex at infinity, and $L_{0}$ and $L_{1}$ closed Lagrangian submanifolds that intersect transversely and $N_{L_{j}} \geq 3$ for $j=0,1$. For the moment, the Lagrangian submanifolds do not have to be monotone. As above, we assume that $L_{0}$ and $L_{1}$ do not intersect the image of the embedded ball. Finally we also assume that there exists a $\omega$-compatible almost complex structure $J$ in $\mathcal{J}_{\text {reg }}\left(L_{0}, L_{1}\right)$ such that $\iota^{*} J=J_{0}$.

Proposition 5.1. Let $(M, \omega), L_{0}, L_{1}$ and $\iota:\left(B^{2 n}(\rho), \omega_{0}\right) \rightarrow(M, \omega)$ as above. If $J$ is a regular $\omega$-compatible almost complex structure for $\left(L_{0}, L_{1}\right)$ and $\widetilde{J}$ the unique $\widetilde{\omega}_{\rho^{-}}$ compatible almost complex structure on $\left(\widetilde{M}, \widetilde{\omega}_{\rho}\right)$ such that $\pi$ is $(\widetilde{J}, J)$-holomorphic, then $\widetilde{J}$ is regular for $\left(\widetilde{L}_{0}, \widetilde{L}_{1}\right)$.

Proof. Let $\widetilde{u}:(D, \partial D) \rightarrow\left(\widetilde{M}, \widetilde{L}_{0} \cup \widetilde{L}_{1}\right)$ be a $\widetilde{J}$-holomorphic disk that joints the intersection points $\widetilde{p}$ and $\widetilde{q}$. Since the blow up map is holomorphic, $\pi \circ \widetilde{u}$ is a $J$ holomorphic disk that joints the intersection points $p=\pi(\widetilde{p})$ and $q=\pi(\widetilde{q})$ and its boundary lies in $L_{0} \cup L_{1}, \pi \circ \widetilde{u}(\cdot, j) \in L_{j}$ for $j=1,2$. Since $J$ is regular for $\left(L_{0}, L_{1}\right)$, then the operator

$$
D_{\bar{\partial}(J), \pi \circ \widetilde{u}}: W_{k}^{p}\left((\pi \circ \widetilde{u})^{*} T M ; L_{0}, L_{1}\right) \rightarrow L_{p}\left((\pi \circ \widetilde{u})^{*} T M\right)
$$

is surjective.

The blow up map induces an operator between the spaces of sections $L_{p}\left(\widetilde{u}^{*} T \widetilde{M}\right)$ and $L_{p}\left((\pi \circ \widetilde{u})^{*} T M\right)$ as follows. In the case when $\widetilde{u}$ does not intersect the exceptional divisor, the map

$$
\pi_{\widetilde{u}}^{L}: L_{p}\left(\widetilde{u}^{*} T \widetilde{M}\right) \rightarrow L_{p}\left((\pi \circ \widetilde{u})^{*} T M\right)
$$

is defined as $\pi_{\widetilde{u}}^{L}(\xi):=\pi_{*}(\xi)$. Note that it is well defined and surjective. Now in the case when $\widetilde{u}(D) \cap E$ is not empty, then since $\widetilde{u}$ is holomorphic we have that $\widetilde{u}^{-1}(E)$ is a finite set in $D$. So in this case $\pi_{\widetilde{u}}^{L}(\xi)$ is defined in the same way as in the previous case on $D \backslash \widetilde{u}^{-1}(E)$ and equal to zero on $\widetilde{u}^{-1}(E)$. Also in this case $\pi_{\widetilde{u}}^{L}$ is well defined and surjective. That is, for every $\widetilde{u}$ holomorphic disk the map $\pi_{\widetilde{u}}^{L}$ is surjective. The same reasoning shows that the map

$$
\pi_{\widetilde{u}}^{W}: W_{p}^{k}\left(\widetilde{u}^{*} T \widetilde{M} ; \widetilde{L}_{0}, \widetilde{L}_{1}\right) \rightarrow W_{p}^{k}\left((\pi \circ \widetilde{u})^{*} T M ; L_{0}, L_{1}\right)
$$

defined as $\pi_{\widetilde{u}}^{W}(\xi)=\pi_{*}(\xi)$ on $D \backslash \widetilde{u}^{-1}(E)$ and zero on $\widetilde{u}^{-1}(E)$ is well defined and surjective.

Notice that we have a commutative relation

$$
\pi_{\widetilde{u}}^{L} \circ D_{\bar{\partial}(\widetilde{J}), \widetilde{u}}=D_{\bar{\partial}(J), \pi \circ \widetilde{u}} \circ \pi_{\widetilde{u}}^{W} .
$$


Since $D_{\bar{\partial}(J), \pi \circ \widetilde{u}}$ is surjective, then $D_{\bar{\partial}(\widetilde{J}), \widetilde{u}}$ is surjective and $\widetilde{J}$ is regular for $\left(\widetilde{L}_{0}, \widetilde{L}_{1}\right)$.

A similar argument applies to the case of regularity of the almost complex structure in the case of the pearl complex.

For $\widetilde{p}$ and $\widetilde{q}$ in $\widetilde{L}_{0} \cap \widetilde{L}_{1}, J$ a regular $\omega$-compatible almost complex structure on $(M, \omega)$ and $\beta \in \pi_{2}\left(\widetilde{M}, \widetilde{L}_{0} \cup \widetilde{L}_{1}\right)$ there is a smooth map

$$
\mathcal{M} \pi: \mathcal{M}(\widetilde{p}, \widetilde{q}, \beta, \widetilde{J}) \rightarrow \mathcal{M}\left(p, q, \pi_{*}(\beta), J\right)
$$

induced by the blow up map. This map is not necessarily surjective. For, suppose that $u$ a $J$-holomorphic disk such that $[u]=\pi_{*}(\beta), \pi_{*}(\beta) \in \pi_{2}\left(M, L_{0} \cup L_{1}\right)$ is non trivial and $u\left(z_{0}\right)=x_{0}$ for some $z_{0} \in \operatorname{Int}(D)$. Then by Propositions 4.1 and 4.6, $u$ has the unique holomorphic lift $\widetilde{u}$ is such that $\mu_{\widetilde{L}_{0}, \widetilde{L}_{1}}(\widetilde{u})<\mu_{L_{0}, L_{1}}(u)$. Hence if the class $\beta$ does not have an exceptional part, we get that the lift $\widetilde{u}$ does not lie in $\mathcal{M}(\widetilde{p}, \widetilde{q}, \beta, \widetilde{J})$.

However if we ignore the homotopy class and consider the whole moduli space, then by Propositions 4.1 and 5.1, the map $\mathcal{M} \pi$ is surjective.

Proposition 5.2. Let $L_{1}$ and $L_{2}$ as in Proposition 5.1. The map

$$
\mathcal{M} \pi: \mathcal{M}(\widetilde{p}, \widetilde{q}, \widetilde{J}) \rightarrow \mathcal{M}(p, q, J)
$$

given by $\mathcal{M} \pi(\widetilde{u})=\pi \circ \widetilde{u}$ is surjective.

Next we give the proof of some of the results that where stated at the Introduction which are related to Lagrangian Floer homology.

Theorem 1.2. Let $L_{0}, L_{1} \subset(M, \omega)$ be admissible Lagrangian submanifolds. Assume that there exists $J \in \mathcal{J}_{\text {reg }}\left(L_{0}, L_{1}\right)$ such that $\iota^{*} J=J_{0}$ where $\iota:\left(B^{2 n}(\rho), \omega_{0}\right) \rightarrow(M, \omega)$ is a symplectic embedding that avoids the Lagrangian submanifolds and $\rho$ is given by Eq. (11). If $\pi_{j}:\left(\widetilde{M}_{j}, \widetilde{\omega}_{j}\right) \rightarrow(M, \omega)$ are the monotone one-point blow up of $(M, \omega)$ at $x_{j} \in M \backslash\left(L_{0} \cup L_{1}\right)$ for $j=1,2$, then

$$
\mathrm{HF}_{*}\left(\pi_{1}^{-1}\left(L_{0}\right), \pi_{1}^{-1}\left(L_{1}\right)\right) \simeq \mathrm{HF}_{*}\left(\pi_{2}^{-1}\left(L_{0}\right), \pi_{2}^{-1}\left(L_{1}\right)\right)
$$

as $\Lambda$-modules.

Proof. The one-point blow up is defined on symplectic manifolds of dimension greater than two. Therefore there exists a path connecting $x_{1}$ to $x_{2}$ that does not intersect the Lagrangian submanifolds. Hence there is a Hamiltonian diffeomorphism $\psi$ on $(M, \omega)$ such that $\psi\left(x_{1}\right)=x_{2}$ and its support does not intersects $L_{0} \cup L_{1}$.

Since the image of $\iota:\left(B^{2 n}(\rho), \omega_{0}\right) \rightarrow(M, \omega)$ misses the Lagrangian submanifolds, then $\psi \circ \iota_{1}$ is also a symplectic embedding of the ball such that $\psi \circ \iota(0)=x_{2}$ and its image also misses the Lagrangians. For $j=1,2$, let $\left(\widetilde{M}_{j}, \widetilde{\omega}_{j}\right)$ be the corresponding symplectic one-point blow up at $x_{j}$. Note that $\left(\psi^{-1}\right)^{*}(J)$ is regular and $(\psi \circ \iota)^{*} \circ\left(\psi^{-1}\right)^{*}(J)=J_{0}$. Then by Proposition 5.1, $J$ and $\left(\psi^{-1}\right)^{*}(J)$ induced regular almost complex structure on the their respective one-point blow up. Moreover, $J$-holomorphic strips that go thru $x_{1}$ are mapped to $\left(\psi^{-1}\right)^{*}(J)$-holomorphic 
strips that go thru $x_{2}$ preserving the Maslov-Viterbo index and vice versa. Hence $\mathrm{HF}_{*}\left(\pi_{1}^{-1}\left(L_{0}\right), \pi_{1}^{-1}\left(L_{1}\right)\right)$ in $\left(\widetilde{M}_{1}, \widetilde{\omega}_{1}\right)$ is isomorphic as $\Lambda$-modules to $\mathrm{HF}_{*}\left(\pi_{2}^{-1}\left(L_{0}\right), \pi_{2}^{-1}\left(L_{1}\right)\right)$ in $\left(\widetilde{M}_{2}, \widetilde{\omega}_{2}\right)$.

The Floer differential $\partial: \mathrm{CF}\left(L_{0}, L_{1}\right) \rightarrow \mathrm{CF}\left(L_{0}, L_{1}\right)$ only involves holomorphic strips of Maslov-Viterbo index 1. From Proposition 4.6, we know that a holomorphic strip of Maslov-Viterbo index 1 in $(M, \omega)$ lifts also to a holomorphic strip of index 1 in $\left(\widetilde{M}, \widetilde{\omega}_{\rho}\right)$. But also, from Proposition 4.6, a holomorphic strip in $(M, \omega)$ of MaslovViterbo index $2 n-1$ lift also to a holomorphic strip of Maslov-Viterbo index 1. Further if $J$ is regular for $\left(L_{0}, L_{1}\right)$, from Proposition 5.1 we know that $\widetilde{J}$ is also regular. Therefore if on $M \backslash\left(L_{0} \cup L_{1}\right)$ there exists a point that is not contained in a $J$-holomorphic strip of Maslov-Viterbo index $2 n-1$, then

$$
\operatorname{HF}_{*}\left(\widetilde{L}_{0}, \widetilde{L}_{1}\right) \simeq \operatorname{HF}_{*}\left(L_{0}, L_{1}\right)
$$

This arguments proves the following proposition of the Introduction.

Theorem 1.3. Let $L_{0}, L_{1} \subset(M, \omega)$ be admissible Lagrangians. If there exists $x_{0} \in$ $M \backslash\left(L_{0} \cup L_{1}\right)$ and $J \in \mathcal{J}_{\text {reg }}\left(L_{0}, L_{1}\right)$ such that $\iota^{*} J=J_{0}$; where $\iota$ is the symplectic embedding of the ball, and $x_{0}$ does not lie in any J-holomorphic strip of MaslovViterbo index $2 n-1$, then

$$
\operatorname{HF}_{*}\left(\widetilde{L}_{0}, \widetilde{L}_{1}\right) \simeq \operatorname{HF}_{*}\left(L_{0}, L_{1}\right)
$$

\section{Proof of Theorem 1.4 And Computation of $\mathrm{QH}_{*}\left(\widetilde{\mathbb{T}}_{\mathrm{Cliff}}\right)$}

First we give a proof of Theorem 1.4 .

Theorem 1.4. Let $L$ be an admissible Lagrangian in $(M, \omega)$ that is $(1,2)$-uniruled of order $2, x_{0} \in M \backslash L$ a generic point and suppose that $\operatorname{dim}(M)=4$. If $p$ and $q$ in $L$ are critical points of a generic Morse-Smale function $f$ with respect to a generic Riemannian metric $g$ on $L$ such that $\operatorname{ind}(p)-\operatorname{ind}(q)-1=-2$, then

$$
\langle\widetilde{d}(p), q\rangle=\langle d(p), q\rangle+\mathbb{Z}_{2} k
$$

where $k$ is the number, mod 2 , of classes $A \in \mathrm{H}_{2}(M, L ; \mathbb{Z})$ such that for some $J \in$ $\mathcal{J}_{\text {reg }}(M, L)$, such that $\iota^{*} J=J_{0}$ where $\iota$ is the symplectic embedding of the ball, the moduli space of pearly trajectories $\mathcal{P}(p, q, A ; g, f, J)$ is non empty, $\mu_{L}(A)=4$ and there is a J-holomorphic disk $u$ such that $x_{0} \in u(D)$ and $A=[u]$.

Proof. Since the Lagrangian is admissible, there is an embedding $\iota:\left(B^{4}(\rho), \omega_{0}\right) \rightarrow$ $(M \backslash L, \omega)$ such that $\iota(0)=x_{0}$ and $\iota^{*} J=J_{0}$ for some $J \in \mathcal{J}_{\text {reg }}(M, L)$. Further by Proposition [5.1, the induced almost complex structure $\widetilde{J}$ in $\left(\widetilde{M}, \widetilde{\omega}_{\rho}\right)$ is regular. Thus $\widetilde{J}$-holomorphic disk with boundary in $\widetilde{L}$ map under the blow up map to $J$-holomorphic disk with boundary in $L$, and vice versa. 
In order to compute $\langle\widetilde{d}(p), q\rangle$ where $p$ and $q$ in $L=\widetilde{L} \subset\left(\widetilde{M}, \widetilde{\omega}_{\rho}\right)$ are given as in the statement, we must count all pearly trajectories in $\left(\widetilde{M}, \widetilde{\omega}_{\rho}\right)$ that go from $p$ to $q$ that contain a single $\widetilde{J}$-holomorphic disk of Maslov index 2. By Proposition 4.6, a pearly trajectory in $(M, \omega)$ that goes from $p$ to $q$ that contains a single $J$-holomorphic disk of Maslov index 2 lifts to a desire pearly trajectory in $\left(\widetilde{M}, \widetilde{\omega}_{\rho}\right)$. Note that since the Maslov index is two, generically the image of the $(D, \partial D)$ under the whole collection of $J$-maps in such a class is 3-dimensional in $M$. Thus, generically it avoids the generic point $x_{0}$. Moreover if the pearly trajectory contains a $J$-holomorphic disk in the class $A \in \mathrm{H}_{2}(M, L)$, the lift contains a $\widetilde{J}$-holomorphic disk in the class $\widetilde{A} \in \mathrm{H}_{2}(\widetilde{M}, \widetilde{L})$.

Again by Proposition 4.6, it follows that a pearly trajectory in $(M, \omega)$ that goes from $p$ to $q$ that contains a single $J$-holomorphic disk of Maslov index 4, say in the class $A$, and moreover there is a $J$-holomorphic disk such that $x_{0} \in u(D)$ and $A=[u]$, lifts to a desire pearly trajectory in $\left(\widetilde{M}, \widetilde{\omega}_{\rho}\right)$. That is, to a pearly trajectory in $\left(\widetilde{M}, \widetilde{\omega}_{\rho}\right)$ from $p$ to $q$ that contains a single $\widetilde{J}$-holomorphic disk of Maslov index 2 in the class $\widetilde{A}-L_{E}$.

Note that this are the only possibilities of pearly trajectories in $\left(\widetilde{M}, \widetilde{\omega}_{\rho}\right)$. Hence the theorem follows.

As an application of our results we compute the pearly differential of $\widetilde{\mathbb{T}}_{\text {Cliff }} \subset$ $\left(\widetilde{\mathbb{C} P^{2}}, \widetilde{\omega}_{\rho}\right)$. Recall from the work of Fukaya, Oh, Ohta and Ono [11]; and Entov and Polterovich in [6], that $\widetilde{\mathbb{T}}_{\text {Cliff }}$ is a wide Lagrangian. We present an alternative approach of this fact.

Theorem 1.5. After blowing up one point the proper transform of the Clifford torus $\mathbb{T}_{\text {Cliff }} \subset\left(\mathbb{C} P^{2}, \omega_{\mathrm{FS}}\right)$ in $\left(\widetilde{\mathbb{C} P}{ }^{2}, \widetilde{\omega}_{\rho}\right)$ is also a wide Lagrangian.

Proof. Assume that $\mathbb{T}_{\text {Cliff }}$ is equipped with a generic Riemannian structure and a Morse-Smale function $f$ with four critical points $p_{0}, p_{1}^{a}, p_{1}^{b}$ and $p_{2}$. Here the index notation in the critical point indicates the Morse index of the critical point. It is know from [4] that the standard complex structure $J$ on $\left(\mathbb{C} P^{2}, \omega_{\mathrm{FS}}\right)$ is regular and that the pearl differential $d$ is identically zero.

Further in order to compute the pearl differential $\widetilde{d}$ on the one-point blow up, we need to know the pearly trajectories that contribute to $d$. To that end, consider the generators $A_{0}, A_{1}, A_{2} \in \mathrm{H}_{2}\left(\mathbb{C} P^{2}, \mathbb{T}_{\text {Cliff }} ; \mathbb{Z}\right)$ such that $A_{1}$ and $A_{2}$ when restricted to $\mathbb{T}_{\text {Cliff }}$ go to $c_{1}$ and $c_{2}$ respectively and generate $\mathrm{H}_{1}\left(\mathbb{T}_{\text {Cliff }} ; \mathbb{Z}\right)$. Thus, $A_{0}+A_{1}+A_{2}$ represents the absolute class of the line in $\mathrm{H}_{2}\left(\mathbb{C} P^{2}, \mathbb{T}_{\text {Cliff }} ; \mathbb{Z}\right)$ and $\mu_{\mathbb{T}_{\text {Cliff }}}\left(A_{0}\right)=\mu_{\mathbb{T}_{\text {Cliff }}}\left(A_{1}\right)=$ $\mu_{\mathbb{T}_{\text {Cliff }}}\left(A_{2}\right)=2$.

According to the dimension of the moduli space of pearly trajectories there are exactly four instances where the pearly trajectories are formed by non constant $J$ holomorphic disks. Namely $\left\langle d\left(p_{0}\right), p_{1}^{a}\right\rangle,\left\langle d\left(p_{0}\right), p_{1}^{b}\right\rangle,\left\langle d\left(p_{1}^{a}\right), p_{2}\right\rangle$ and $\left\langle d\left(p_{1}^{b}\right), p_{2}\right\rangle$. Moreover, in each case there are exactly two the pearly trajectories each containing a single 
$J$-holomorphic disk of Maslov index 2. For example, there are two pearly trajectories from $p_{0}$ to $p_{1}^{a},\left\langle d\left(p_{0}\right), p_{1}^{a}\right\rangle=0$. Both trajectories start with a $J$-holomorphic disk thru $p_{0}$ follow by a flow line that goes to $p_{1}^{a}$. In one case the $J$-holomorphic disk lies in the class $A_{0}$ and in the other case we can assumed to be $A_{1}$. That is we assume that the class $c_{1} \in \mathrm{H}_{1}\left(\mathbb{T}_{\text {Cliff }} ; \mathbb{Z}\right)$ intersects the unstable submanifold at $p_{1}^{a}$. For the other three cases the pearly trajectories that appear are similar to the case the was described.

Additionally let $x_{0}=[1: a: b]$ be a generic point in $\mathbb{C} P^{2} \backslash \mathbb{T}_{\text {Cliff }}$ such that $0<|a|<|b|<1$. Hence $\widetilde{\mathbb{C} P^{2}}$ will stand for the blow up of $\mathbb{C} P^{2}$ at $x_{0}$. Moreover by [4, Cor. 1.2.5] there is a symplectic embedding of $\left(B^{4}(1 / \sqrt{3}), \omega_{0}\right)$ into $\left(\widetilde{\mathbb{C} P}{ }^{2} \backslash \widetilde{\mathbb{T}}_{\text {Cliff }}, \omega_{\mathrm{FS}}\right)$. Hence by Lemmas 4.5 and $4.4, \widetilde{\mathbb{T}}_{\text {Cliff }} \subset\left(\widetilde{\mathbb{C} P^{2}}, \widetilde{\omega}_{\rho}\right)$ is a monotone Lagrangian submanifold with minimal Maslov number equal to 2. Henceforth, the Lagrangian quantum homology of $\widetilde{\mathbb{T}}_{\text {Cliff }} \subset\left(\widetilde{\mathbb{C} P}{ }^{2}, \widetilde{\omega}_{\rho}\right)$ is well defined.

Recall that $\left(\mathbb{C} P^{2}, \omega_{\mathrm{FS}}\right)$ is $(1,1)$-uniruled of order 4 . Hence we cannot conclude directly that $\mathrm{QH}_{*}\left(\mathbb{T}_{\text {Cliff }}\right)$ and $\mathrm{QH}_{*}\left(\widetilde{\mathbb{T}}_{\text {Cliff }}\right)$ are isomorphic. In order to compute $\tilde{d}$ and $\mathrm{QH}_{*}\left(\widetilde{\mathbb{T}}_{\text {Cliff }}\right)$ we use the same Riemannian structure and Morse-Smale function on the Lagrangian submanifold. Since $J$ is a complex structure on $\mathbb{C} P^{2}$, then it induces a complex structure $\widetilde{J}$ on $\widetilde{\mathbb{C} P}{ }^{2}$. Further, by Proposition $[5.1, \widetilde{J}$ is regular for $\left(\widetilde{\mathbb{C} P}{ }^{2}, \widetilde{\mathbb{T}}_{\text {Cliff }}\right)$.

Now we compute the pearly trajectories in $\widetilde{\mathbb{C} P}{ }^{2}$ that connect $p_{0}$ to $p_{1}^{a}$ and show that $\left\langle\widetilde{d}\left(p_{0}\right), p_{1}^{a}\right\rangle=0$. More precisely, the value of $k$ of Theorem 1.4 is two. The two pearly trajectories described above still exist; each having $\widetilde{J}$-holomorphic disks in the classes $\widetilde{B}$ and $\widetilde{A}_{1}$. Note that $\mu_{\widetilde{\mathbb{T}}_{\text {Cliff }}}(\widetilde{B})=\mu_{\widetilde{\mathbb{T}}_{\text {Cliff }}}\left(\widetilde{A}_{1}\right)=2$. However there are two new pearly trajectories that are induced by $J$-holomorphic disks in $\left(\mathbb{C} P^{2}, \mathbb{T}_{\text {Cliff }}\right)$ with Maslov index 4 . For this, we rely on the classification of $J$-holomorphic disks on $\left(\mathbb{C} P^{2}, \mathbb{T}_{\text {Cliff }}\right)$ given by Cho in [5, Theorem 10.1]. There are six classes of $J$-holomorphic disk with Maslov index $4, A_{j}+A_{k}$ for $j, k \in\{0,1,2\}$. Of those only the classes $A_{1}+A_{2}$ and $A_{0}+A_{2}$ contain $J$-holomorphic disks that go thru $x_{0}$ and when restricted to $\mathbb{T}_{\text {Cliff }}$ intersect the unstable submanifold at $p_{1}^{a}$. Hence the new pearly trajectories that contribute to $\left\langle\widetilde{d}\left(p_{0}\right), p_{1}^{a}\right\rangle$ star with a $\widetilde{J}$-holomorphic disk thru $p_{0}$ in the class $\widetilde{A}_{1}+\widetilde{A}_{2}-L_{E}$ and a flow line to $p_{1}^{a}$; the other also stars with a $\widetilde{J}$-holomorphic disk thru $p_{0}$ in the class $\widetilde{A}_{0}+\widetilde{A}_{2}-L_{E}$ and a flow line to $p_{1}^{a}$. Hence $\left\langle\widetilde{d}\left(p_{0}\right), p_{1}^{a}\right\rangle=0$.

A similar argument applies to the other cases to conclude that $\left\langle\widetilde{d}\left(p_{0}\right), p_{1}^{b}\right\rangle=\left\langle\widetilde{d}\left(p_{1}^{a}\right), p_{2}\right\rangle=$ $\left\langle\widetilde{d}\left(p_{1}^{b}\right), p_{2}\right\rangle=0$. Hence $\mathrm{QH}_{*}\left(\widetilde{\mathbb{T}}_{\text {Cliff }}\right)=\mathrm{H}_{*}\left(\widetilde{\mathbb{T}}_{\text {Cliff }} ; \mathbb{Z}_{2}\right) \otimes \Lambda$ and $\widetilde{\mathbb{T}}_{\text {Cliff }}$ is a wide Lagrangian in $\left(\mathbb{C} P^{2}, \widetilde{\omega}_{\rho}\right)$.

\section{REFERENCES}

[1] Auroux, D. A beginner's introduction to Fukaya categories. In Contact and symplectic topology, vol. 26 of Bolyai Soc. Math. Stud. János Bolyai Math. Soc., Budapest, 2014, pp. 85-136. 
[2] Biran, P. Lagrangian barriers and symplectic embeddings. Geom. Funct. Anal. 11, 3 (2001), 407-464.

[3] Biran, P., And Cornea, O. A Lagrangian quantum homology. In New perspectives and challenges in symplectic field theory, vol. 49 of CRM Proc. Lecture Notes. Amer. Math. Soc., Providence, RI, 2009, pp. 1-44.

[4] Biran, P., ANd Cornea, O. Rigidity and uniruling for Lagrangian submanifolds. Geom. Topol. 13, 5 (2009), 2881-2989.

[5] CHO, C.-H. Holomorphic discs, spin structures, and Floer cohomology of the Clifford torus. Int. Math. Res. Not., 35 (2004), 1803-1843.

[6] Entov, M., And Polterovich, L. Rigid subsets of symplectic manifolds. Compos. Math. 145,3 (2009), 773-826.

[7] Floer, A. Morse theory for Lagrangian intersections. J. Differential Geom. 28, 3 (1988), 513547.

[8] Floer, A. Witten's complex and infinite-dimensional Morse theory. J. Differential Geom. 30, 1 (1989), 207-221.

[9] Fukaya, K., Oh, Y.-G., Ohta, H., And Ono, K. Lagrangian intersection Floer theory: anomaly and obstruction. Part I, vol. 46 of AMS/IP Studies in Advanced Mathematics. American Mathematical Society, Providence, RI; International Press, Somerville, MA, 2009.

[10] Fukaya, K., Оh, Y.-G., Оhta, H., AND Ono, K. Lagrangian intersection Floer theory: anomaly and obstruction. Part II, vol. 46 of AMS/IP Studies in Advanced Mathematics. American Mathematical Society, Providence, RI; International Press, Somerville, MA, 2009.

[11] Fukaya, K., OH, Y.-G., Ohta, H., And Ono, K. Lagrangian Floer theory on compact toric manifolds. I. Duke Math. J. 151, 1 (2010), 23-174.

[12] McDuff, D., And Polterovich, L. Symplectic packings and algebraic geometry. Invent. Math. 115, 3 (1994), 405-434. With an appendix by Yael Karshon.

[13] McDuff, D., And Salamon, D. Introduction to symplectic topology, second ed. Oxford Mathematical Monographs. The Clarendon Press, Oxford University Press, New York, 1998.

[14] McDuff, D., And Salamon, D. J-holomorphic curves and symplectic topology, second ed., vol. 52 of A. M. S. Colloquium Publications. American Mathematical Society, Providence, RI, 2012.

[15] OH, Y.-G. Floer cohomology of Lagrangian intersections and pseudo-holomorphic disks. I. Comm. Pure Appl. Math. 46, 7 (1993), 949-993.

[16] Он, Y.-G. Addendum to: "Floer cohomology of Lagrangian intersections and pseudoholomorphic disks. I." [Comm. Pure Appl. Math. 46 (1993), no. 7, 949-993; MR1223659 (95d:58029a)]. Comm. Pure Appl. Math. 48, 11 (1995), 1299-1302.

[17] Он, Y.-G. Relative Floer and quantum cohomology and the symplectic topology of Lagrangian submanifolds. In Contact and symplectic geometry (Cambridge, 1994), vol. 8 of Publ. Newton Inst. Cambridge Univ. Press, Cambridge, 1996, pp. 201-267.

Facultad de Ciencias, Universidad de Colima, Bernal Díaz del Castillo No. 340, Colima, Col., Mexico 28045

E-mail address: andres_pedroza@ucol.mx 